\title{
GPPS-BJ-2019-0177
}

\section{Evaluation of Spalart-Allmaras model with various modifications for predicting corner separation in a compressor cascade}

\author{
Wenhao Li ${ }^{2}$, Yangwei Liu ${ }^{1,2^{\star}}$ \\ 1. State Key Laboratory of Aerodynamics, China \\ Aerodynamics Research and Development \\ Center, P.O.BOX 211 \\ Mianyang Sichuan, 621000, China \\ 2. National Key Laboratory of Science and \\ Technology on Aero-Engine Aero- \\ Thermodynamics, School of Energy and Power \\ Engineering, Beihang University \\ Beijing, 100191, China \\ *Corresponding author: liuyangwei@126.com
}

\begin{abstract}
Three-dimensional (3D) corner separation is an inherent flow feature in axial compressor which blocks flowpath and aggravates the performance. Numerical simulation of corner separation flow is conducted in Prescribed Velocity Distribution (PVD) cascade with several versions of SpalartAllmaras (SA) model. Turbulence models studied here include original SA model, SA-neg model, SA-noft2 model, SA-R model, SA-KL model, SA-Helicity model and their combinations with Quadratic Constitutive Relation (QCR) method. Compared with experimental results, the SAHelicity model predicts more accurately than other models at zero incidence. Results provided by most blending models adopting QCR relation are better than that of original SA model, but still deviate from experimental data.
\end{abstract}

\section{INTRODUCTION}

3D corner separation is an inevitable complex flow phenomenon in axial compressor. The separation region in suction surface-endwall corner blocks flowpath and therefore aggravates the load and pressurize performance of compressor. Besides, the mixing of main flow and secondary flow from separation leads to losses and thus brings down the efficiency (Gbadebo, 2005). Consequently, predicting the 3D corner separation flow accurately is significant for flow mechanism study as well as axial compressor designing and optimization.

Although Direct Numerical Simulation (DNS) and Large-Eddy Simulation (LES) have already been applied in scientific research, Reynolds-Averaged Navier-Stokes (RANS) method will still be widely used in decades (Spalart, 2000). Among all RANS models, the one equation SA model (Spalart, 1994) is followed with great interest since its relatively high accuracy, robustness and low calculation cost. However, it has been proved that the SA model overestimates the high-loss region in $3 \mathrm{D}$ corner flow prediction (Scillitoe, 2015). It is essential to conduct improvements on original SA model.

In this paper, CFD code CFL3D is used to simulate the typical 3D corner separation flow in PVD cascade with different versions of SA model, which include original SA model, SA-neg model (Allmaras, 2012), SA-noft2 model (Aupoix, 2003), SA-R model (Dacles-Mariani, 1995), SAKL model (Nichols, 2019), SA-Helicity (Liu, 2011) model and their combinations with QCR2000/QCR2013 method. Numerical results are compared with experimental data in order to obtain well-performed modifications, and therefore giving valuable guidance for scientific research and engineering applications.

\section{METHODOLOGY}

\section{Computational Domain and Boundary conditions}

Designed by Rolls-Royce, the linear PVD cascade (Gbadebo, 2003) based on controlled diffusion airfoil (CDA) is studied in this paper on behalf of high pressure stator blades. The geometrical and inflow parameters for PVD cascade is shown in Table 1.

Table 1 Parameters for PVD Cascade

\begin{tabular}{c|c}
\hline Parameter & Value \\
\hline Chord, $c$ & $0.1515 \mathrm{~m}$ \\
\hline Blade Height/Chord, $h / c$ & 1.32 \\
\hline Blade Pitch/Chord, $s / c$ & 0.926 \\
\hline Blade Thickness/Chord, $t / c$ & 0.1 \\
\hline Blade Camber Angle, $\varphi$ & $42.0^{\circ}$ \\
\hline Blade Stagger Angle, $\gamma$ & $14.7^{\circ}$ \\
\hline Inlet Blade Angle, $\beta_{1}$ & $41.0^{\circ}$ \\
\hline Inlet Reynolds Number, $R e$ & $2.3 \times 10^{5}$ \\
\hline
\end{tabular}

3D separation flow under zero incidence is simulated since its typicality. Besides, different turbulence models show various results at this incidence. With about 1.5 million 
grids in half of span, an $\mathrm{O} 4 \mathrm{H}$ type grid generated by Numeca/Autogrid5 is shown in Figure 1. The height of the first grid line to solid wall is set to $y^{+} \approx 0.7$.

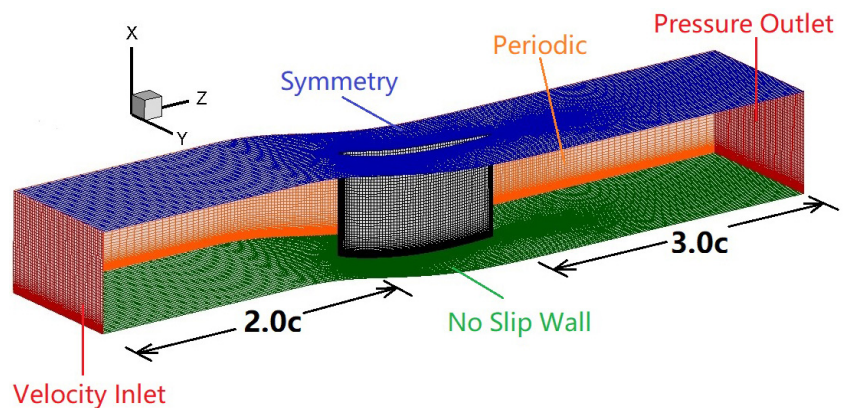

Figure 1 Mesh Topology and Boundary Conditions

As for the boundary conditions, velocity profile from experiment is specified at the inlet. The main flow velocity is $23.82 \mathrm{~m} / \mathrm{s}$, and the turbulent viscosity ratio to molecular viscosity is set to 50 . Back pressure at the pressure outlet is 101325Pa. As in Figure 1, a symmetry is implied to reduce calculation costs.

\section{Numerical Method}

Simulations in this work are carried out using opensource code CFL3D (Krist, 1998) which is a structured-grid, Finite Volume Method based RANS flow solver. The code is modified for internal-flow simulation and more turbulence models are added as alternatives. In all cases presented in this work, third-order upwind-biased differencing spatial scheme and first-order implicit time scheme are applied.

\section{Governing Equations}

In code CFL3D, three-dimensional compressible NavierStokes equations are solved. As for turbulence models, various modifications of original SA model are referred whose modelling equation is:

$$
\begin{aligned}
\frac{\partial \hat{v}}{\partial t}+ & u_{j} \frac{\partial \hat{v}}{\partial x_{j}}=c_{b 1}\left(1-f_{t 2}\right) \hat{S} \hat{v}-\left[c_{w 1} f_{w}-\frac{c_{b 1}}{\kappa^{2}} f_{t 2}\right]\left(\frac{\hat{v}}{d}\right)^{2} \\
& +\frac{1}{\sigma}\left[\frac{\partial}{\partial x_{j}}\left((v+\hat{v}) \frac{\partial \hat{v}}{\partial x_{j}}\right)+c_{b 2} \frac{\partial \hat{v}}{\partial x_{i}} \frac{\partial \hat{v}}{\partial x_{i}}\right]
\end{aligned}
$$

where

$$
\hat{S}=\Omega+\frac{\hat{v}}{\kappa^{2} d^{2}} f_{v 2}
$$

Based on original SA model, the SA-neg model aims at the non-physical situation when $\hat{v}<0$ appears somewhere in flow field. The model is the same as SA model under most circumstances. However, when the turbulence variable $\hat{v}<$ 0 , the turbulence equation turns into:

$$
\begin{gathered}
\frac{\partial \hat{v}}{\partial t}+u_{j} \frac{\partial \hat{v}}{\partial x_{j}}=c_{b 1}\left(1-c_{t 3}\right) \Omega \hat{v}+c_{w 1}\left(\frac{\hat{v}}{d}\right)^{2} \\
+\frac{1}{\sigma}\left[\frac{\partial}{\partial x_{j}}\left(\left(v+\hat{v} f_{n}\right) \frac{\partial \hat{v}}{\partial x_{j}}\right)+c_{b 2} \frac{\partial \hat{v}}{\partial x_{i}} \frac{\partial \hat{v}}{\partial x_{i}}\right]
\end{gathered}
$$

The SA-noft 2 model fixes $f_{t 2}$ to zero on the basis of original SA model. Parameter $f_{t 2}$ is originally implied to make zero a stable solution in order to slightly delay the unexpected transition caused by numerical problems (Spalart, 1994). When there is no transition in the flow field $\left(\hat{v}_{\infty} \geq 3 v_{\infty}\right.$, where $v_{\infty}$ stands for molecular viscosity in the farfield) or the trip term is omitted, $f_{t 2}$ is not necessary.

Based on SA model, the SA-R model reduces $\hat{S}$ in regions where the magnitude of vorticity $\Omega$ exceeds the strain rate $S$. This modification reflects the physics mechanism that turbulence production should be weakened in rigidly rotation regions. The $\hat{S}$ term gets replaced by:

$$
\hat{S}=\Omega+C_{\text {rot }} \min (0, S-\Omega)+\frac{\hat{v}}{\kappa^{2} d^{2}} f_{v 2} \quad C_{\text {rot }}=2.0
$$

Similar to SA-R, the SA-KL model also reduces $\hat{S}$ in regions where the magnitude of vorticity exceeds strain rate, such as in quasi rigidly rotational regions. The modification makes no difference where vorticity is close to strain rate, such as in thin shear layers. The SA-R model mentioned above and the SA-KL model are considered as less efficient but much simpler versions of SA-RC model (Rumsey, 2019). In this model, the $\hat{S}$ term is written as:

$$
\hat{S}=\sqrt{S \Omega}+\frac{\hat{v}}{\kappa^{2} d^{2}} f_{v 2}
$$

Based on SA-noft2, the SA-Helicity model brings in the influence of energy backscatter. It is proved that the velocity helicity is correlated with energy backscatter and therefore acts an important role in model modifications (Liu, 2011). The $\hat{S}$ term is modified to increase turbulence production according to the value of relative helicity density $h$ :

where

$$
\hat{S}=\left(1+C_{h 1} h^{C_{h 2}}\right) \Omega+\frac{\hat{v}}{\kappa^{2} d^{2}} f_{v 2}
$$

$$
h=\frac{\left|\omega_{i} u_{i}\right|}{\Omega U} \quad \omega_{i}=\varepsilon_{i j k} \frac{\partial u_{j}}{\partial x_{k}} \quad U=\sqrt{u_{i} u_{i}}
$$

The constants are $C_{h 1}=0.71$ and $C_{h 2}=0.6$.

Unlike all the models mentioned above, SA-QCR2000 model (Spalart, 2000) reserves the original form of SA turbulence model equation but the Reynolds stresses are calculated by:

$$
\tau_{i j, Q C R}=\tau_{i j}-C_{c r 1}\left[O_{i k} \tau_{j k}+O_{j k} \tau_{i k}\right]
$$

where

$$
O_{i k}=2 W_{i k} / \sqrt{\frac{\partial u_{m}}{\partial x_{n}} \frac{\partial u_{m}}{\partial x_{n}}} \quad W_{i k}=\frac{1}{2}\left(\frac{\partial u_{i}}{\partial x_{k}}-\frac{\partial u_{k}}{\partial x_{i}}\right)
$$

Since turbulent kinetic energy $k$ is not available in SA model, the SA-QCR2013 model (Mani, 2013) is improved based on SA-QCR2000, with an additional term to normal stresses which acts similarly as the $-2 \rho k / 3$ term in Boussinesq relation.

$$
\begin{gathered}
\tau_{i j, Q C R}=\tau_{i j}-C_{c r 1}\left[O_{i k} \tau_{j k}+O_{j k} \tau_{i k}\right] \\
-C_{c r 2} \mu_{t} \sqrt{2 S_{m n}^{*} S_{m n}^{*}} \delta_{i j}
\end{gathered}
$$

where

$$
S_{i j}^{*}=S_{i j}-\frac{1}{3} \frac{\partial u_{k}}{\partial x_{k}} \delta_{i j} \quad C_{c r 2}=2.5
$$




\section{RESULTS AND DISCUSSION}

In comparison with experimental data, outflow angle, surface static pressure distribution, loss coefficient and relative displacement thickness are calculated from simulation results.

\section{Results from Commonly Used Models}

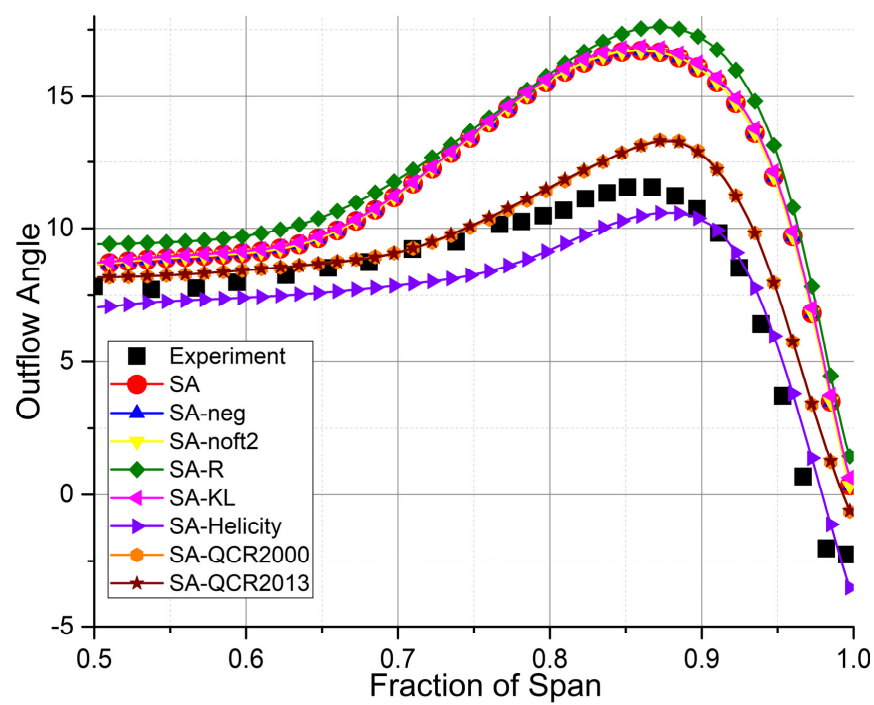

Figure 2 Mass Averaged Outflow Angle (Commonly Used Models)

Firstly, results from different linear eddy-viscosity models (EVMs) including SA, SA-neg, SA-noft2, SA-R, SA$\mathrm{KL}$ and SA-Helicity are analysed together with QCR models (SA-QCR2000, SA-QCR2013). These forms of turbulence model are cited from publications and are commonly used. At $50 \%$ of axial chord from the trailing edge, the spanwise variation of mass weighted averaged outflow angle is studied among simulation results and experiment. As shown in Figure 2, the corner flow of PVD cascade is thoroughly turbulence thus SA-neg model and SA-noft2 model show no difference from original SA model. Overall, SA model overestimates the flow angle at the outlet, and SA-R model distracts more from the experimental data. The result of SA$\mathrm{KL}$ model is similar to that of SA model but slightly worse. SA-Helicity and SA-QCR models give better predictions than original SA model.

The accuracy of simulation near the middle of span represents the performance of turbulence models in terms of quasi-3D flow prediction. SA-QCR2000, SA-QCR2013 and SA-Helicity model perform the best in this region, with SAQCR2000 and SA-QCR2013 slightly larger than experiment and SA-Helicity slightly lower. SA-KL model which overestimates the outflow angle shows nearly no difference from SA model. SA-R model performs even worse than original SA model.

The influence of 3D separation gets larger from $70 \%$ to $100 \%$ of span. Boundary layer at suction surface of the blade is much thicker, and thus the main flow is lift up away from the blade which leads to larger outflow angle. The accuracy of simulation in this region represents the performance of turbulence models in terms of 3D separation flow prediction.
SA-Helicity predicts the best near the root of blade $(90 \%$ to $100 \%$ of span) which proves that increasing the turbulence production according to flow field quantity such as the helicity density is necessary in 3D separation simulations. As non-linear models, SA-QCR2000 and SA-QCR2013 give similar results which are better than original SA model. But their difference from experiment from $80 \%$ to $100 \%$ of span shows that the modification to Boussinesq relation is not adequate in $3 \mathrm{D}$ corner separation prediction.

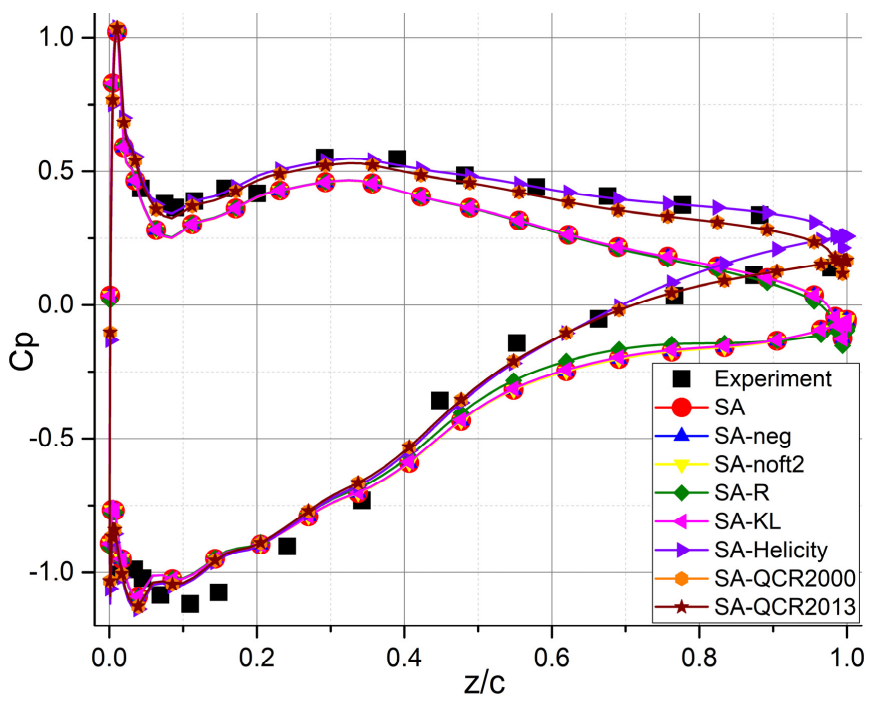

Figure 3 Surface Static Pressure (54\% of span, Commonly Used Models)

The nondimensional variable $\mathrm{Cp}$ which represents the forces between fluid and solid wall is calculated from surface static pressure. Accurately predicting the $\mathrm{Cp}$ helps analysing forces on the blades and the influence of blades to fluid. The expression of $\mathrm{Cp}$ is:

$$
C_{p}=\frac{P_{01}-P}{P_{01}-P_{1}}
$$

where $P_{01}$ stands for reference total pressure at the inlet, $P_{1}$ is the reference static pressure, $P$ is the local static pressure.

Figure 3 shows the surface static pressure distribution at $54 \%$ of span. On the whole, except for the region from $0 \%$ to $40 \%$ of chord on the suction side where all models show no difference, SA, SA-R and SA-KL model get much lower Cp than experimental data. Compared with SA model, the SA-R model shows a slightly higher $\mathrm{Cp}$ around $60 \%$ of chord on the suction side. SA-neg and SA-noft2 still have same results as original SA model. SA-Helicity and SA-QCR models perform well in general, but all get lower pressure difference between pressure and suction sides of blade than experiment. On the pressure side of blade, SA-Helicity fits the best with experimental datum. On the suction side, the SA-QCR models give accurate prediction of $\mathrm{Cp}$ from $60 \%$ to $100 \%$ of chord. Once again, two QCR models get similar results.

As for $89 \%$ of span shown in Figure 4, the conclusions are generally similar to that from $54 \%$ of span. Differently, the results of SA-QCR models are not good enough especially on the suction surface, which meet the verdict mentioned before that QCR models have defectiveness at the region near the root of blade. The SA-Helicity model 
behaves the best on the suction side, however it slightly over predicts the $\mathrm{Cp}$ near trailing edge on both sides.

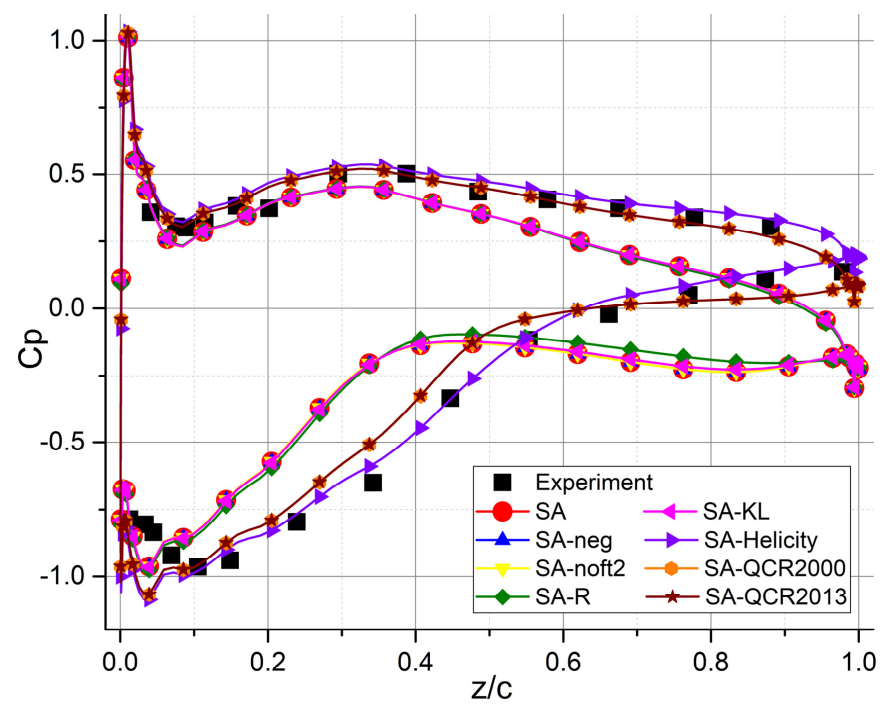

Figure 4 Surface Static Pressure (89\% of span, Commonly Used Models)

3D corner separation contributes to a large part of flow loss in compressor and it is significant to be accurately predicted during compressor design and optimization. In our work, the total pressure loss coefficient is implied to describe the flow loss:

$$
\text { Total Pressure Loss }=\frac{P_{01}-P_{0}}{P_{01}-P_{1}}
$$

where $P_{0}$ stands for local total pressure.

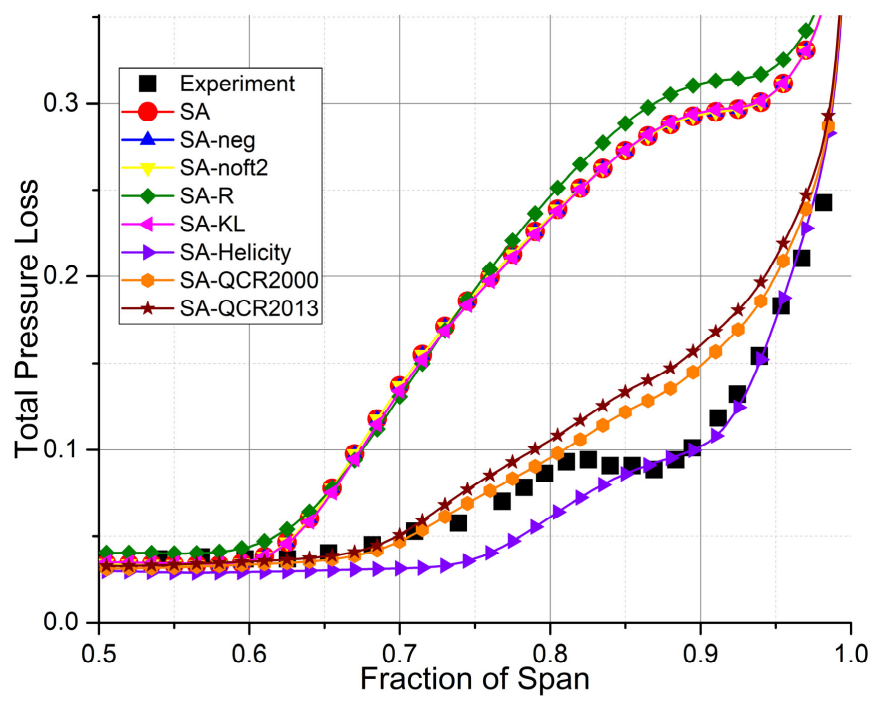

Figure 5 Mass Averaged Total Pressure Loss (Commonly Used Models)

Figure 5 shows the spanwise variation of pitchwise mass averaged total pressure loss coefficient. The SA-R and SA$\mathrm{KL}$ corrections reduce the eddy viscosity production in regions where the magnitude of vorticity $\Omega$ exceeds strain rate $S$. However, the SA-KL model shows no improvements in terms of total pressure loss while SA-R model gives even worse prediction, which indicates that these two modifications are not effective in 3D corner separation simulation. SA-QCR models come into smaller separation region than SA model, but they still over predict the total pressure loss. Relatively, the result given by SA-QCR2000 is slightly better in contrast with that of SA-QCR2013. Comparatively speaking, the result of SA-QCR2000 model fits the best with the experiment from $50 \%$ to $80 \%$ of span. SA-Helicity model also gets similar result as experimental data especially near the root of blade.

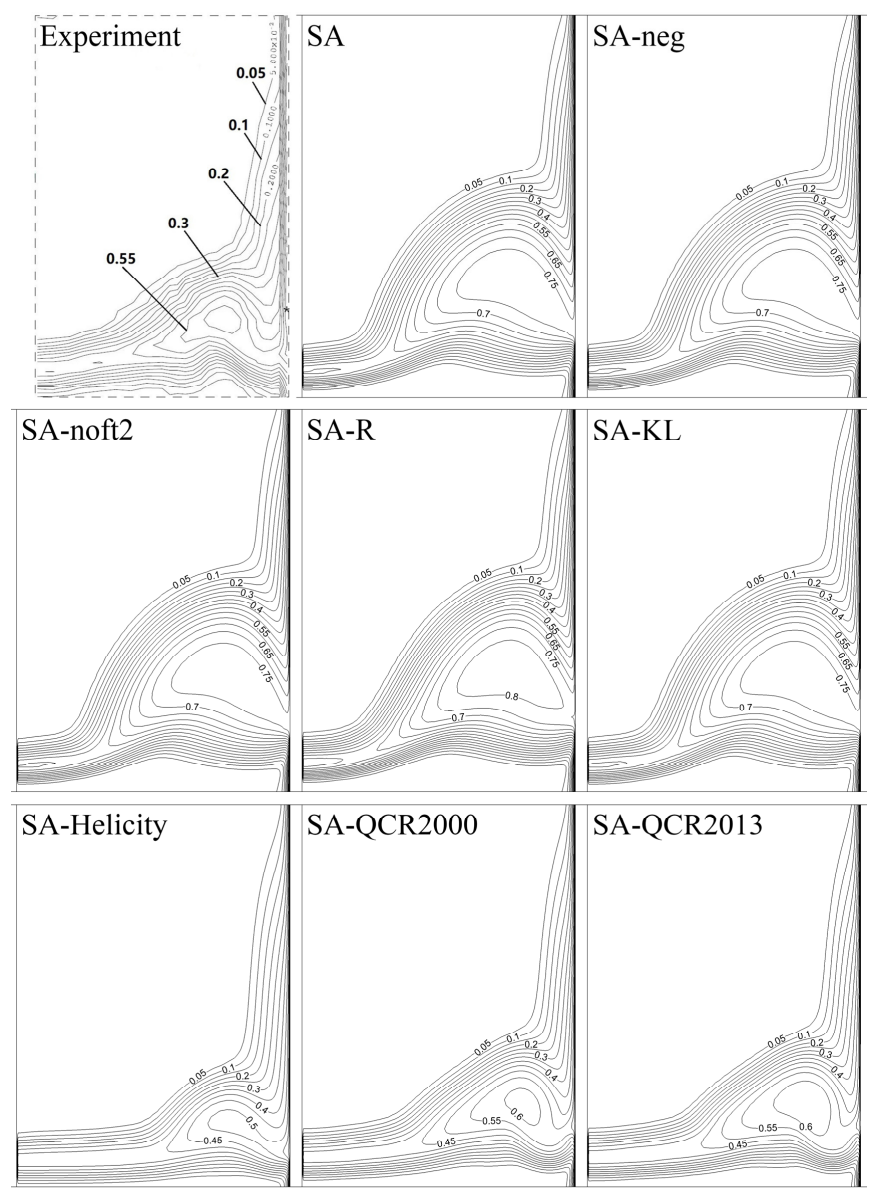

\section{Figure 6 Total Pressure Loss Coefficient (Commonly Used Models)}

Figure 6 shows the total pressure loss coefficient distribution at $50 \%$ of axial chord from the trailing edge. Among all the models, SA-Helicity and SA-QCR models accurately simulate the area of 3D corner separation region. The peak value from SA-Helicity model is lower than the experiment, which results into lower loss coefficient from $65 \%$ to $85 \%$ of span (Figure 5). The peak value given by SAQCR2000 and SA-QCR2013 is close to that of experimental data. However, the high loss region from SA-QCR models are closer to the endwall, which result in higher total pressure loss around $90 \%$ of span (Figure 5). Relatively, SAQCR2013 predicts a little bit worse than SA-QCR2000. As for other models, SA-neg, SA-noft2 and SA-KL model get same results as SA model which obviously over predicts the corner separation region and the total pressure loss coefficient value. The area of corner separation given by SA$\mathrm{R}$ is similar to that of original SA model, but the peak value at core is about $7 \%$ larger. 
The relative displacement thickness at the trailing edge is calculated to represent the thickness of separation region from the suction side of blade. It stands for the size of separation at particular fraction of span. The definition is the difference of the boundary layer displacement thickness between a certain spanwise position and the mid-span:

$$
\begin{gathered}
\left(\delta^{*}(x)-\delta^{*}{ }_{\text {midspan }}\right) / c \\
\delta^{*}(x)=\int_{0}^{\delta}\left[1-\frac{\rho v_{z}(x, y)}{\rho_{f s} V_{z} f s}\right] d y \\
\delta_{\text {midspan }}^{*}=\int_{0}^{\delta}\left[1-\left(\frac{\rho v_{z}(y)}{\rho_{f s} V_{z f s}}\right)_{\text {midspan }}\right] d y
\end{gathered}
$$

where $y$ is the pitchwise distance from the suction surface, $v_{z}$ is the axial velocity and $f s$ stands for free-stream values.

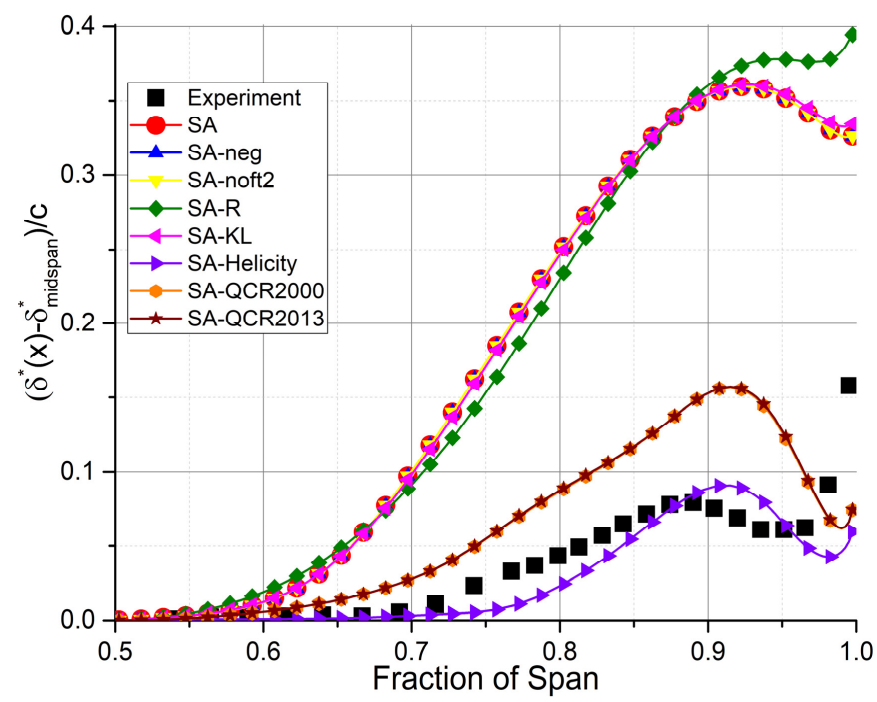

Figure 7 Relative Displacement Thickness (Commonly Used Models)

Figure 7 shows the relative displacement thickness calculated by different models. In terms of this parameter, SA (SA-neg, SA-noft2), SA-KL and SA-R deviate from the experiment datum, the QCR models perform better and the SA-Helicity model has the most accurate prediction.

Considering the experimental data, the relative displacement thickness grows gradually from $65 \%$ to $88 \%$ of span and reaches a maximum value. Then it decreases a little and increases rapidly from $95 \%$ to $100 \%$ of span. As for SAHelicity model, the relative displacement thickness grows later, and gets to the peak at $91 \%$ of span. Its peak value is a little larger than the experiment. The results from SA-QCR models grow earlier and faster than experimental data, and their peak value is one time larger. Other models get much too distant results from the experiment. The relative displacement thickness from SA-R model is slightly lower than SA around $80 \%$ of span but reaches a higher peak value near the blade root.

\section{Models Using QCR Method}

In this section, models which combine EVMs (SA-R, SA-KL and SA-Helicity) and QCR method (QCR2000 and QCR2013) are tested with the same case. As different QCR versions of same EVM show similar results in terms of outflow angle, static pressure distribution and relative displacement thickness, the results from QCR2013 models are displayed as representation.

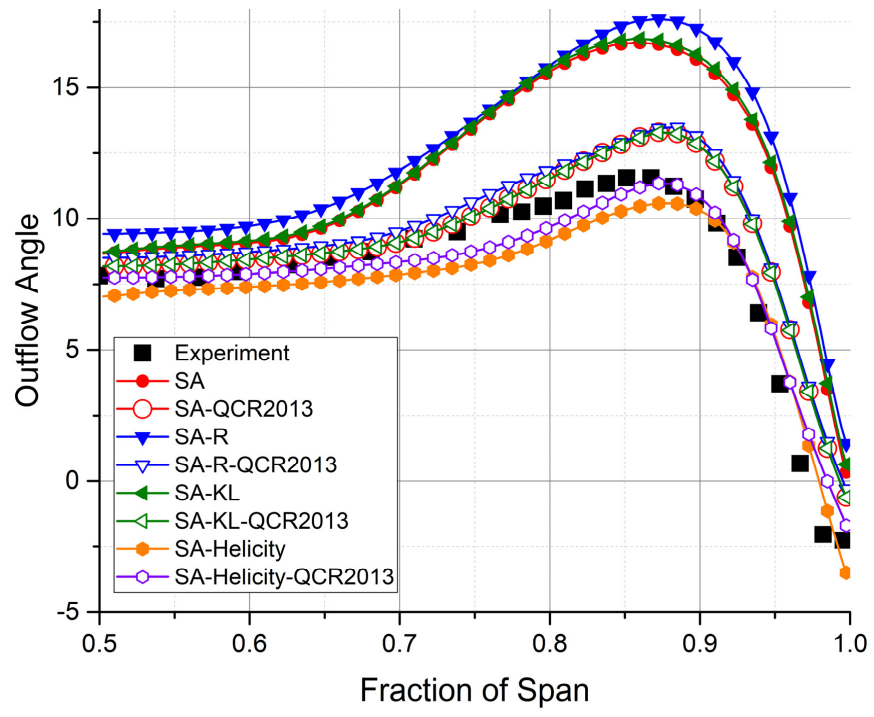

Figure 8 Mass Averaged Outflow Angle (Blending Models)

Figure 8 shows the outflow angle obtained from different blending models. The SA-R-QCR2013, SA-KLQCR2013 and SA-Helicity-QCR2013 models which deserve special focus all received better outcome than original SA model. Specifically, just as SA-R get worse result than SA, SA-R-QCR2013 comes into slightly larger outflow angle than SA-QCR2013. SA-KL-QCR2013 receives same result as the SA-QCR2013 model. The SA-Helicity model receives better result when combined with QCR. The blending model inherits the accuracy of SA-Helicity model at the region from $90 \%$ to $100 \%$ of span, and predicts well at the mid-span. The SA-Helicity-QCR2013 model is the best model in predicting outflow angle on the whole.

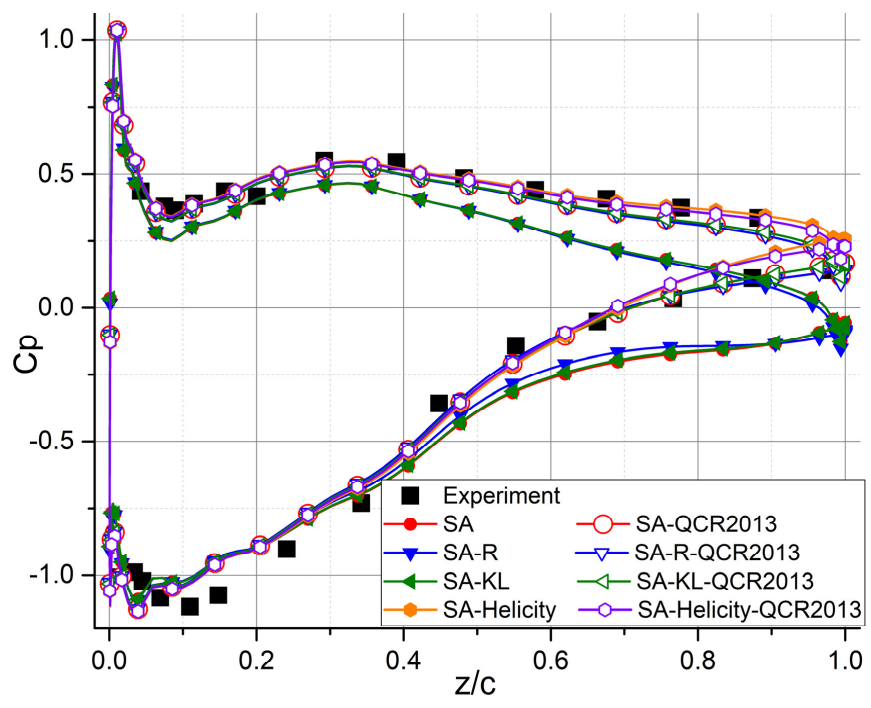

Figure 9 Surface Static Pressure (54\% of span, Blending Models)

In static pressure distribution prediction (Figure 9 and 10), the SA-R-QCR2013 model gets similar result as SA- 
QCR2013 at both 54\% and 89\% of span. As in outflow angle prediction, the SA-KL-QCR2013 model performs the same as SA-QCR2013. Improvement can be observed when applying QCR2013 to SA-Helicity model, since its better agreement with the experimental data near the trailing edge. In Figure 9 and 10, the static pressure distribution of SAHelicity-QCR2013 model on both sides from $80 \%$ to $100 \%$ of chord length fits better with the experiment than the SAHelicity model.

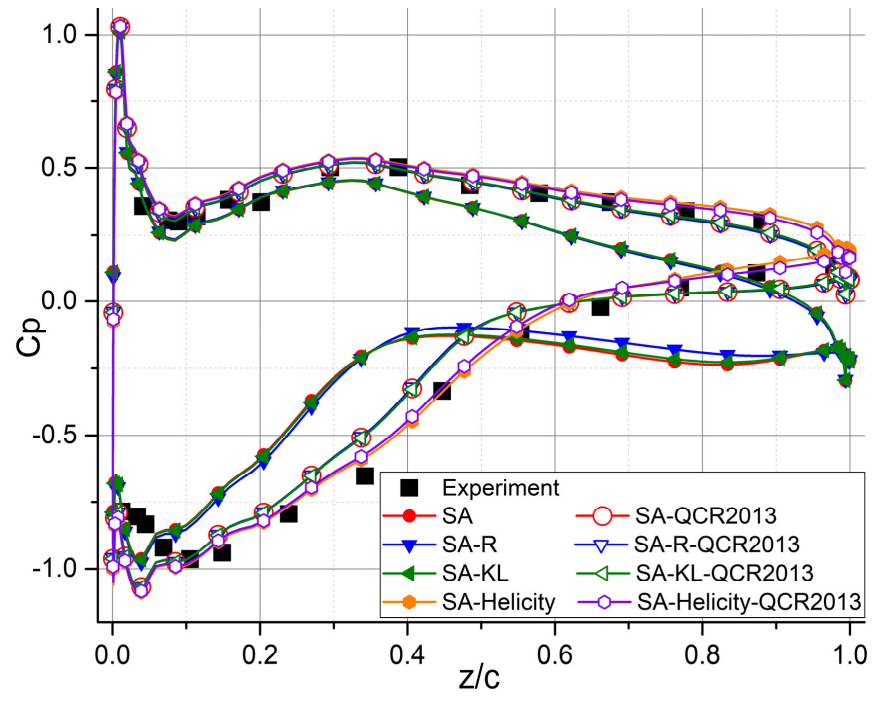

Figure 10 Surface Static Pressure $(89 \%$ of span, Blending Models)

QCR2000 and QCR2013 versions of same EVM show different results in total pressure loss coefficient, thus results from both versions are presented here. As shown in Figure 11, apart from SA-Helicity-QCR2000/2013, other blending models tend to approach similar total pressure loss as which SA-QCR2000 and SA-QCR2013 model get. They all over predict the separation region and the total pressure loss. Among all the blending models, SA-Helicity-QCR2013 model gives the best prediction in terms of loss coefficient. It even performs better than SA-Helicity model.

It also can be found that the blending models inherit features from its components. Consistent with pure SA-QCR models, the QCR2013 version of each EVM gets higher total pressure loss than its QCR2000 version. Besides, it has been shown that SA-R model gets higher total pressure loss than SA-KL model. Then the SA-R-QCR2000/2013 model performs worse than corresponding SA-KL-QCR2000/2013 model here. Similarly, SA-Helicity model underestimates the total pressure loss from $70 \%$ to $80 \%$ of span. As a result, the derivatives SA-Helicity-QCR2000 and SA-HelicityQCR2013 models show the same characteristic.

As for the loss coefficient contour shown in Figure 12, SA-R-QCR2000/2013 and SA-KL-QCR2000/2013 models overestimate the size and peak value of high loss region while SA-Helicity-QCR2000/2013 underestimate it. For each EVM, its QCR2013 blending model gives similar separation structure as its QCR2000 blending model but with slightly higher loss coefficient. As shown in Figure 11 and 12, the SA-R-QCR2000/2013 models over predict the loss coefficient of corner separation region near the root of blade.
And the area of the high loss region is larger than that of SAQCR2000/2013 models. The corner separation structure and the total pressure loss given by SA-KL-QCR2000/2013 models are the same as that of SA-QCR2000/2013 models. SA-Helicity-QCR2013 fits the best with experimental data in both the shape of corner separation region and the peak value.

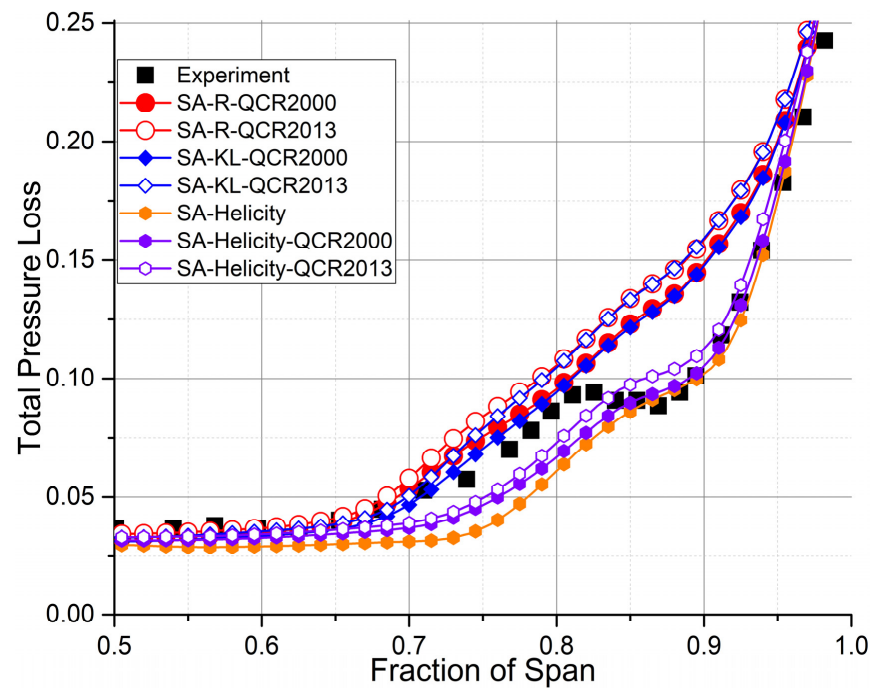

Figure 11 Mass Averaged Total Pressure Loss (Blending Models)

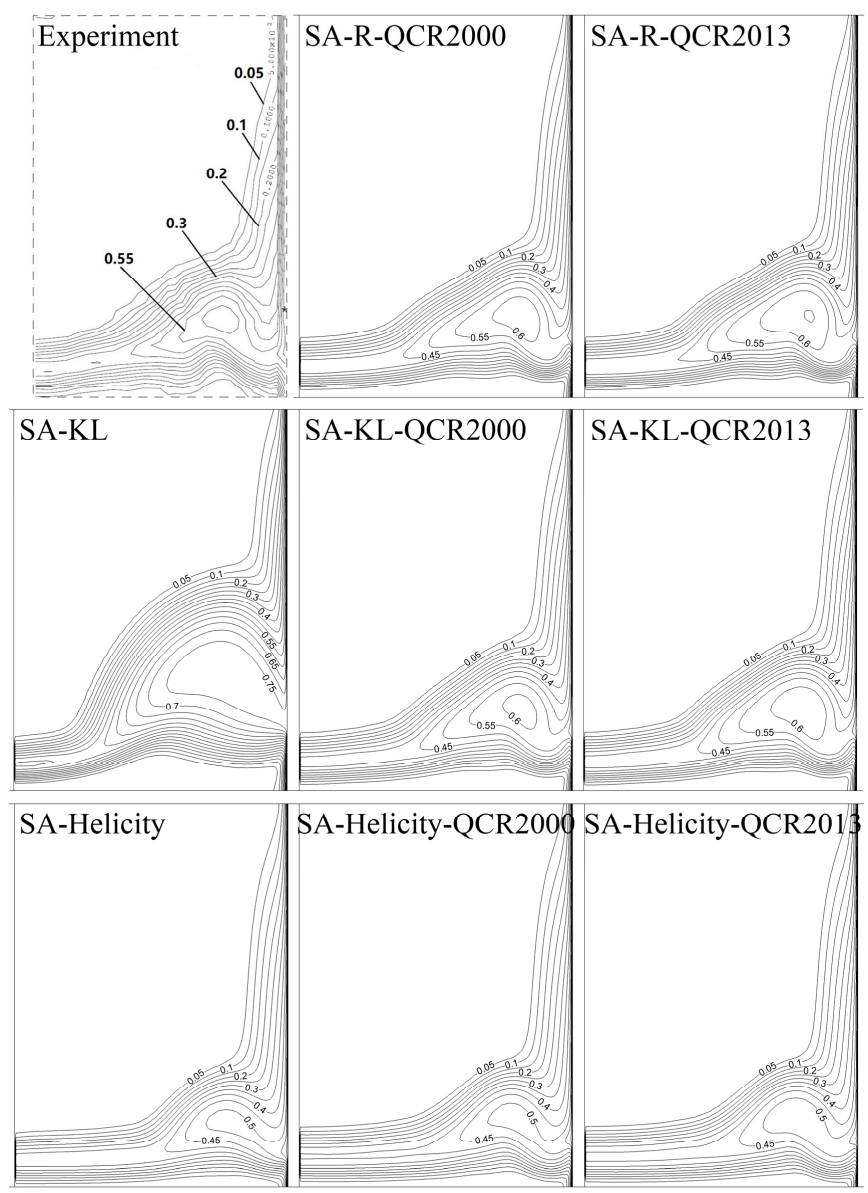

Figure 12 Total Pressure Loss Coefficient (Blending Models) 
Figure 13 shows the relative displacement thickness distribution of various blending models using QCR2013 method along the spanwise. The QCR2000 version of each EVM comes into same result as its QCR2013 version. The SA-QCR2013, SA-R-QCR2013 as well as SA-KL-QCR2013 model show improvement on original SA model but still not good enough. Compared with SA-Helicity model, SAHelicity-QCR2013 model fits better with experiment from $70 \%$ to $85 \%$ of span. In addition, its peak value of relative displacement thickness appears at about $90 \%$ of span which is nearer to the experimental data. In terms of relative displacement thickness, SA-Helicity-QCR2013 model performs the best among all models.

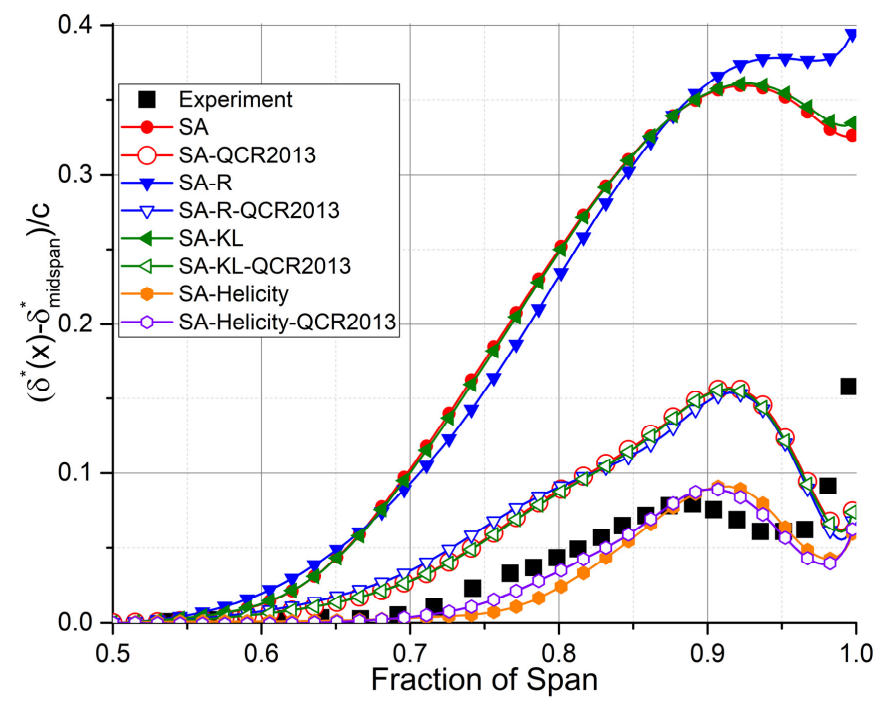

Figure 13 Relative Displacement Thickness (Blending Models)

\section{CONCLUSIONS}

The main conclusions from this research are as follows:

1. The corner separation flow in PVD cascade at 0 incidence predicted by original SA model is much larger than that in experimental data. The modified models show various results with different details from each other.

2. Among commonly used models, SA-Helicity model has the most accurate result. SA-QCR2000 and SAQCR2013 also show visible improvements. SA-neg, SAnoft2, SA-R and SA-KL receive similar results as original SA model.

3. With similar idea, SA-R model and SA-KL model aim to reduce the turbulence production in regions where rotation exceeds shearing effect. The result indicates that these two modifications show nearly no improvements in complex 3D separation flow prediction.

4. After applying QCR method to EVMs, blending models gather the features of component models. Apart from SA-Helicity based models, blending models get similar results to pure QCR models, which are superior to original SA model but not effective enough. SA-Helicity-QCR models especially SA-Helicity-QCR2013 perform better than SA-Helicity and predict the corner separation flow most correctly.

\section{ACKNOWLEDGMENTS}

We express our appreciation to the late Prof. Lipeng Lu, whose contribution to this work was of great significance. This work is supported by the National Natural Science Foundation of China (No. 51676007, No. 51420105008 and No. 51790513), and also supported by the Open fund from State Key Laboratory of Aerodynamics. In addition, the authors are sincerely grateful to Whittle Laboratory and Rolls-Royce plc for their data on PVD cascade.

\section{References}

Allmaras, S. R., Johnson, F. T., and Spalart, P. R. (2012). Modifications and Clarifications for the Implementation of the Spalart-Allmaras Turbulence Model. ICCFD7-1902, 7th International Conference on Computational Fluid Dynamics, Big Island, Hawaii, 9-13 July 2012.

Aupoix, B. and Spalart, P. R. (2003). Extensions of the Spalart-Allmaras turbulence model to account for wall roughness. International Journal of Heat \& Fluid Flow, 24(4):454-462.

Dacles-Mariani, J., Zilliac, G. G., Chow, J. S., and Bradshaw, P. (1995). Numerical/Experimental Study of a Wingtip Vortex in the Near Field. AIAA Journal, Vol. 33, No. 9, pp. 1561-1568.

Gbadebo, S. A. (2003). Three-Dimensional Separations in Compressors. Ph.D. thesis, University of Cambridge, UK.

Gbadebo, S. A., Cumpsty, N. A., and Hynes, T. P. (2005). Three-Dimensional Separations in Axial Compressors. Asme Journal of Turbomachinery 127.2:457-469.

Krist, S. L., Biedron, R. T., and Rumsey, C. L. (1998). CFL3D User's Manual (Version 5.0). NASA TM-1998208444.

Liu, Y., Lu, L., Fang, L., and Gao, F. (2011). Modification of Spalart-Allmaras model with consideration of turbulence energy backscatter using velocity helicity. Physics Letters A, 375(24):2377-2381.

Mani, M., Babcock, D., Winkler, C., and Spalart, P. R. (2013). Predictions of a Supersonic Turbulent Flow in a Square Duct. Aiaa Aerospace Sciences Meeting Including the New Horizons Forum and Aerospace Exposition.

Nichols, R. H., "A Summary of the Turbulence Models in the CREATE $^{\mathrm{TM}}$-AV Kestrel Flow Solvers," AIAA Paper 20191342, January 2019.

Rumsey, C. L. (2019). https://turbmodels.larc.nasa.gov/. Last updated 13 February 2019.

Scillitoe, A. D., Tucker, P. G., and Adami, P. (2015). Evaluation of RANS and ZDES Methods for the Prediction of Three-Dimensional Separation in Axial Flow Compressors. ASME Turbo Expo 2015: Turbine Technical Conference and Exposition: V02BT39A041.

Spalart, P. R. (2000). Strategies for turbulence modelling and simulations. International Journal of Heat \& Fluid Flow, 21(3):252-263.

Spalart, P. R. and Allmaras, S. R. (1994). A one-equation turbulence model for aerodynamic flows. Recherche Aerospatiale, 1(1):5-21. 